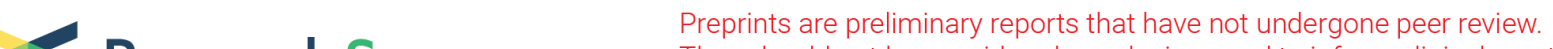 $\begin{array}{ll}\text { Research Square } & \text { They should not be considered conclusive, used to inform clinical practice, } \\ \text { or referenced by the media as validated information. }\end{array}$
}

\section{Decreased Eosinophil Counts and Elevated Lactate Dehydrogenase Predict Severe COVID-19 Patients with Underlying Chronic Airway Diseases}

\section{Lingling Yi}

Tongji Hospital of Tongji Medical College of Huazhong University of Science and Technology

Dian Chen

Tongji Hospital of Tongji Medical College of Huazhong University of Science and Technology

Shuchen Zhang

Tongji Hospital of Tongji Medical College of Huazhong University of Science and Technology

\section{Yuchen Feng}

Tongji Hospital of Tongji Medical College of Huazhong University of Science and Technology

\section{Wenliang Wu}

Tongji Hospital of Tongji Medical College of Huazhong University of Science and Technology

\section{Chenli Chang}

Tongji Hospital of Tongji Medical College of Huazhong University of Science and Technology

\section{Shengchong Chen}

Tongji Hospital of Tongji Medical College of Huazhong University of Science and Technology

Guohua Zhen ( $\nabla$ ghzhen@tjh.tjmu.edu.cn )

Tongji Hospital of Tongji Medical College of Huazhong University of Science and Technology

\section{Research}

Keywords: Eosinopenia, Predictor, Chronic airway inflammation, COVID-19

Posted Date: August 10th, 2020

DOI: https://doi.org/10.21203/rs.3.rs-53471/v1

License: (1) (1) This work is licensed under a Creative Commons Attribution 4.0 International License. Read Full License 


\section{Abstract}

Background: Several predictors for the severity of coronavirus disease 2019 (COVID-19) have been reported, including decreased circulating lymphocytes and eosinophil counts. However, chronic airway inflammation characterized by accumulated lymphocytes or eosinophils may affect the pathogenesis of COVID-19. We aimed to investigate the predictors for the severity of COVID-19 in patients with chronic airway diseases.

Methods: In this retrospective cohort study, we reviewed medical records of all laboratory-confirmed COVID-19 patients with chronic bronchitis, chronic obstructive pulmonary disease (COPD) and asthma admitted in Sino-French New City Branch of Tongji Hospital, a large regional hospital in Wuhan, China, from January $26^{\text {th }}$ to April $3^{\text {rd }}$. The Tongji Hospital ethics committee approved this study.

Results: There were 59 patients with underlying chronic airway inflammation including chronic bronchitis, COPD, and asthma. When compared with non-severe patients, severe patients were more likely to have decreased lymphocyte counts ( 0.6 vs. $1.1 \times 10 \otimes / L, p<0.001)$, eosinopenia $(<0.02 \times 10 \otimes / L, 73 \%$ vs. $24 \%, p$ $<0.001)$, increased lactate dehydrogenase (LDH) (471.0 vs. $230.0 \mathrm{U} / \mathrm{L}, p<0.001)$ and elevated IL-6 level (47.4 vs. $5.7 \mathrm{pg} / \mathrm{ml}, p=0.002$ ) on admission. Eosinopenia and elevated LDH were significantly associated with disease severity in both univariate and multivariate regression models included the above variables. Eosinopenia was also an independent risk factor for mortality of this cohort in a multivariate model included the above variables. Moreover, eosinophil counts and LDH levels tended to return to normal range over time in both groups after treatment and severe patients recovered slower than non-severe patients, especially eosinophil counts.

Conclusions: Eosinopenia and elevated LDH are potential predictors of disease severity in COVID-19 patients with underlying chronic airway diseases. Theses predictors may help clinicians identify the severe COVID-19 patients with chronic bronchitis, COPD, and asthma.

\section{Introduction}

In December 2019, coronavirus disease 2019 (COVID-19) caused by severe acute respiratory syndrome coronavirus-2 (SARS-CoV-2) occurred in Wuhan, Hubei Province, China. As of July 20, 2020, 14348858 of COVID-19 confirmed cases and 603691 deaths were reported in over 215 countries worldwide [1], demanding an urgent need for early identification for severe cases. The SARS-CoV-2 virus, which belongs to the betacoronavirus, is highly homologic (with $88 \%$ identity) to two bat-derived SARS-like coronaviruses, while more distant from SARS-CoV (around 79\%) and Middle East respiratory syndrome coronavirus (MERS-CoV, around 50\%) [2]. Clinical evidence has suggested that SARS-CoV can be transmitted from person to person via direct contact or through droplets from infected individuals [3, 4]. SARS-CoV- 2 is able to attack the respiratory system through binding the cell entry receptors angiotensinconverting enzyme 2 (ACE2) on airway epithelial cells and results in pneumonia and respiratory failure in critically ill patients. 
Chronic bronchitis, chronic obstructive pulmonary disease (COPD), and asthma are common respiratory diseases with chronic airway inflammation [5-9]. Eosinophils, neutrophils, and macrophages in innate immune response significantly increase in the airway and lung during the initial phase of inflammation. Subsequently, activated adaptive immunity leads to the recruitment of T and B lymphocytes. Th1, Th2, and Th17 cells play a crucial role in COPD, asthma, and chronic bronchitis, resulting in mucus overproduction and airflow obstruction [5, 8]. Lymphocytopenia, however, has been reported in several studies in severe patients infected with SARS-CoV-2 [10-12]. Recently, circulating eosinophil counts were also reported to be decreased in COVID-19 patients, and associated with the severity of the disease [13, 14]. Therefore, patients with underlying COPD, asthma, and chronic bronchitis may have different inflammatory states after SARS-CoV-2 infection compared to patients without chronic airway inflammation. [10-12, 15-17]. In this study, we aimed to identify the potential predictors for the disease severity of COVID-19 patients with underlying chronic airway diseases including chronic bronchitis, COPD, and asthma.

In this retrospective cohort study, we reviewed medical records of 59 laboratory-confirmed COVID-19 patients with underlying chronic airway inflammation and compared the demographic, clinical, and radiological characteristics as well as laboratory results between severe and non-severe patients in this cohort. Potential predictors of disease severity were identified in the abnormal laboratory findings using univariate and multivariate regression models.

\section{Results}

\section{Demographics and clinical characteristics of non-severe and severe COVID-19 patients with chronic airway diseases}

A total of 1888 patients were admitted. Fifty-nine patients with underlying chronic airway inflammation, including COPD $(0.95 \%)$, asthma $(0.53 \%)$, and chronic bronchitis $(1.64 \%)$ were confirmed to have SARSCoV-2 infection. Thirty-three patients were classified as non-severe patients and twenty-six patients were classified as severe patients. Although COPD was more common in severe COVID-19 patients when compared with non-severe COVID-19 patients ( $42 \%$ vs. $21 \%$ ), the difference was not statistically significant.

The median age for all patients was 71 years (interquartile range, 57-80) and more than half of them (54\%) were over 70 years old. The majority $(71 \%)$ of patients were male (Table 1$)$. There was no significant difference in age and sex between non-severe and severe patients. Thirty-one (53\%) patients had one or more comorbidities besides the three chronic airway diseases, with cardiovascular disease (46\%) and endocrine system disease (15\%) being the most common comorbidity. There were no significant differences in the presence of these comorbidities between the non-severe and severe COVID19 patients. Half of the patients had smoking histories or current smokers. 
Table 1

Demographics and clinical characteristics of COVID-19 patients with chronic airway inflammation on admission.

\begin{tabular}{|c|c|c|c|c|}
\hline & $\begin{array}{l}\text { All patients }(n= \\
59)\end{array}$ & $\begin{array}{l}\text { Non-severe patients ( } \mathrm{n} \\
=33 \text { ) }\end{array}$ & $\begin{array}{l}\text { Severe patients ( } n \\
=26 \text { ) }\end{array}$ & $\begin{array}{l}\mathrm{p} \\
\text { value }\end{array}$ \\
\hline Age & $71(57-80)$ & $70(55-79)$ & $74(64-82)$ & 0.184 \\
\hline$<30 \mathrm{yr}$ & $2(3 \%)$ & $1(3 \%)$ & $1(4 \%)$ & 0.726 \\
\hline $30-49 y r$ & $5(8 \%)$ & $4(12 \%)$ & $1(4 \%)$ & \\
\hline $50-69 y r$ & $20(34 \%)$ & $11(33 \%)$ & $9(35 \%)$ & \\
\hline$\geq 70$ year & $32(54 \%)$ & $17(52 \%)$ & 15 (58\%) & \\
\hline \multicolumn{5}{|l|}{ Sex } \\
\hline Female & $17(29 \%)$ & $10(30 \%)$ & $7(27 \%)$ & 0.776 \\
\hline Male & $42(71 \%)$ & $23(70 \%)$ & $19(73 \%)$ & \\
\hline Comorbidity & $31(53 \%)$ & $17(52 \%)$ & $14(54 \%)$ & 0.859 \\
\hline COPD & $18(30 \%)$ & $7(21 \%)$ & $11(42 \%)$ & 0.081 \\
\hline Chronic bronchitis & 31 (53\%) & $18(55 \%)$ & $13(50 \%)$ & 0.728 \\
\hline Asthma & $10(17 \%)$ & $8(24 \%)$ & $2(8 \%)$ & 0.093 \\
\hline $\begin{array}{l}\text { Cardiovascular } \\
\text { Disease }\end{array}$ & $27(46 \%)$ & $14(42 \%)$ & $13(50 \%)$ & 0.562 \\
\hline $\begin{array}{l}\text { Cerebrovascular } \\
\text { Disease }\end{array}$ & $3(5 \%)$ & $1(3 \%)$ & $2(8 \%)$ & 0.418 \\
\hline $\begin{array}{l}\text { Digestive system } \\
\text { Disease }\end{array}$ & $1(2 \%)$ & $0(0 \%)$ & $1(4 \%)$ & 0.441 \\
\hline $\begin{array}{l}\text { Endocrine system } \\
\text { Disease }\end{array}$ & $9(15 \%)$ & $4(12 \%)$ & $5(19 \%)$ & 0.451 \\
\hline Nephrosis & $2(3 \%)$ & $1(3 \%)$ & $1(4 \%)$ & 0.863 \\
\hline $\begin{array}{l}\text { Urinary system } \\
\text { Disease }\end{array}$ & $1(2 \%)$ & $1(3 \%)$ & $0(0 \%)$ & 1.000 \\
\hline Viral hepatitis & $1(2 \%)$ & $1(3 \%)$ & $0(0 \%)$ & 1.000 \\
\hline Smokers & $29(49 \%)$ & $14(42 \%)$ & $15(58 \%)$ & 0.244 \\
\hline
\end{tabular}

Data are median (IQR), $\mathrm{n}(\%)$, or $\mathrm{n} / \mathrm{N}(\%)$, where $\mathrm{N}$ is the total number of patients with available data. $\mathrm{p}$ values comparing severe with non-severe patients were calculated by $\chi 2$ test, Fisher's exact test, or Mann-Whitney U test, as appropriate. COVID-19, coronavirus disease 2019. 


\begin{tabular}{|c|c|c|c|c|}
\hline & $\begin{array}{l}\text { All patients }(n= \\
59)\end{array}$ & $\begin{array}{l}\text { Non-severe patients ( } \mathrm{n} \\
=33 \text { ) }\end{array}$ & $\begin{array}{l}\text { Severe patients ( } n \\
=26 \text { ) }\end{array}$ & $\begin{array}{l}\mathrm{p} \\
\text { value }\end{array}$ \\
\hline \multicolumn{5}{|l|}{ Fever } \\
\hline$<38.5^{\circ} \mathrm{C}$ & $21(36 \%)$ & 13 (39\%) & $8(31 \%)$ & 0.407 \\
\hline$\geq 38.5^{\circ} \mathrm{C}$ & 28 (47\%) & $14(42 \%)$ & $14(54 \%)$ & \\
\hline Cough & 43 (73\%) & $21(64 \%)$ & $22(85 \%)$ & 0.072 \\
\hline Chest tightness & $16(27 \%)$ & $7(21 \%)$ & $9(35 \%)$ & 0.250 \\
\hline Hemoptysis & $1(2 \%)$ & $0(0 \%)$ & $1(4 \%)$ & 0.441 \\
\hline Dyspnea & 25 (42\%) & $8(24 \%)$ & 17 (65\%) & 0.001 \\
\hline Fatigue & $28(47 \%)$ & $15(45 \%)$ & $13(50 \%)$ & 0.728 \\
\hline Myalgia & $11(19 \%)$ & $7(21 \%)$ & $4(15 \%)$ & 0.568 \\
\hline Nausea & $2(3 \%)$ & $1(3 \%)$ & $1(4 \%)$ & 0.863 \\
\hline Anorexia & $2(3 \%)$ & $0(0 \%)$ & $2(8 \%)$ & 0.190 \\
\hline Diarrhea & 15 (25\%) & $9(27 \%)$ & $6(23 \%)$ & 0.713 \\
\hline Headache & $2(3 \%)$ & $0(0 \%)$ & $2(8 \%)$ & 0.190 \\
\hline
\end{tabular}

The most common symptoms were fever (83\%), cough (73\%), fatigue (47\%) and dyspnea (42\%). Dyspnea was more common in severe patients compared to non-severe patients ( $65 \%$ vs. $24 \%, p=0.001)$ (Table 1$)$.

\section{Laboratory findings of non-severe and severe COVID-19 patients with chronic airway diseases}

When compared with non-severe patients, severe patients were more likely to have elevated neutrophil counts ( 8.2 vs. $4.1 \times 10 \varangle / L, p=0.001)$, decreased lymphocyte counts ( 0.6 vs. $1.1 \times 10 \otimes / L, p<0.001)$,

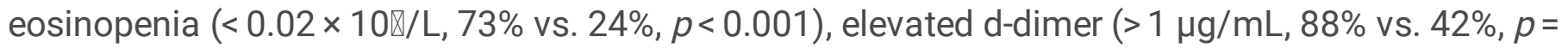
$0.001)$, increased lactate dehydrogenase (LDH) (471.0 vs. $230.0 \mathrm{U} / \mathrm{L}, p<0.001)$, elevated blood urea nitrogen ( $>9.5 \mathrm{mmol} / \mathrm{L}, 42 \%$ vs. $3 \%, p<0.001)$, increased hypersensitive troponin I $(>34 \mathrm{pg} / \mathrm{mL}, 48 \% \mathrm{vs}$. $7 \%, p=0.001$ ), and increased inflammation markers including C-reactive protein (CRP) (126.2 vs. $19.9 \mathrm{mg} / \mathrm{L}, p<0.001$ ), procalcitonin ( $\geq 0.05 \mathrm{ng} / \mathrm{mL}, 96 \%$ vs. $43 \%, p<0.001)$ and ferritin (1264.2 vs. $293.6 \mathrm{mg} / \mathrm{L}, p=0.004$ ) (Table 2). Of note, significant differences in the expression of inflammationrelated cytokines including interleukin (IL)-6, IL-8 and tumor necrosis factor (TNF)-a were observed between the two groups, which were dramatically increased in severe patients. 
Table 2

Laboratory findings of COVID-19 patients with chronic airway inflammation on admission.

\begin{tabular}{|c|c|c|c|c|c|}
\hline & $\begin{array}{l}\text { Normal } \\
\text { range }\end{array}$ & $\begin{array}{l}\text { All patients } \\
(n=59)\end{array}$ & $\begin{array}{l}\text { Non-severe } \\
\text { patients }(n= \\
\text { 33) }\end{array}$ & $\begin{array}{l}\text { Severe } \\
\text { patients }(n= \\
\text { 26) }\end{array}$ & $\begin{array}{l}p \\
\text { value }\end{array}$ \\
\hline $\begin{array}{l}\text { White blood cell count, } \times \\
10 \rrbracket / L\end{array}$ & $\begin{array}{l}4.00- \\
10.00\end{array}$ & $\begin{array}{l}6.66(4.54- \\
9.61)\end{array}$ & $\begin{array}{l}5.98(3.71- \\
7.43)\end{array}$ & $\begin{array}{l}9.27(5.86- \\
12.83)\end{array}$ & 0.002 \\
\hline$<4$ & & $13(22 \%)$ & $9(27 \%)$ & $4(15 \%)$ & 0.004 \\
\hline $4-10$ & & $33(56 \%)$ & $22(67 \%)$ & $11(42 \%)$ & \\
\hline$>10$ & & $13(22 \%)$ & $2(6 \%)$ & $11(43 \%)$ & \\
\hline Neutrophil count, $\times 10 \varangle / \mathrm{L}$ & $\begin{array}{l}1.80- \\
6.30\end{array}$ & $\begin{array}{l}4.50(2.73- \\
7.99)\end{array}$ & $\begin{array}{l}4.12(2.36- \\
5.42)\end{array}$ & $\begin{array}{l}8.16(3.90- \\
12.00)\end{array}$ & 0.001 \\
\hline Lymphocyte count, $\times 10 \rrbracket / L$ & $\begin{array}{l}1.10- \\
3.20\end{array}$ & $\begin{array}{l}0.84(0.53- \\
1.44)\end{array}$ & $\begin{array}{l}1.14(0.80- \\
1.73)\end{array}$ & $\begin{array}{l}0.56(0.28- \\
0.76)\end{array}$ & 0.000 \\
\hline$<1 \cdot 1$ & & $38(64 \%)$ & $16(48 \%)$ & $22(85 \%)$ & 0.004 \\
\hline$\geq 1 \cdot 1$ & & $21(36 \%)$ & $17(52 \%)$ & $4(15 \%)$ & \\
\hline 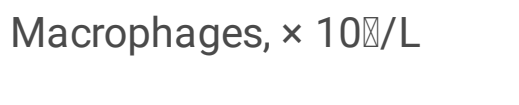 & $\begin{array}{l}0.10- \\
0.60\end{array}$ & $\begin{array}{l}0.51(0.35- \\
0.63)\end{array}$ & $\begin{array}{l}0.51(0.36- \\
0.66)\end{array}$ & $\begin{array}{l}0.52(0.33- \\
0.63)\end{array}$ & 0.891 \\
\hline Eosinophil count, $\times 10 \mathbb{L} / \mathrm{L}$ & $\begin{array}{l}0.02- \\
0.52\end{array}$ & $\begin{array}{l}0.02(0.00- \\
0.08)\end{array}$ & $\begin{array}{l}0.04(0.02- \\
0.11)\end{array}$ & $\begin{array}{l}0.00(0.00- \\
0.02)\end{array}$ & 0.000 \\
\hline$<0.02$ & & $27(46 \%)$ & $8(24 \%)$ & $19(73 \%)$ & 0.000 \\
\hline$\geq 0.02$ & & $32(54 \%)$ & $25(76 \%)$ & $7(27 \%)$ & \\
\hline Basophils, $\times 10 \otimes / L$ & $\begin{array}{l}0.00- \\
0.10\end{array}$ & $\begin{array}{l}0.01(0.01- \\
0.03)\end{array}$ & $\begin{array}{l}0.01(0.01- \\
0.03)\end{array}$ & $\begin{array}{l}0.01(0.01- \\
0.03)\end{array}$ & 0.657 \\
\hline Haemoglobin, g/L & $\begin{array}{l}130.0- \\
175.0\end{array}$ & $\begin{array}{l}129.0(112.0- \\
139.0)\end{array}$ & $\begin{array}{l}134.0(116.5- \\
141.0)\end{array}$ & $\begin{array}{l}125.5(109.0- \\
134.8)\end{array}$ & 0.184 \\
\hline 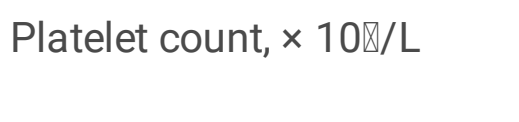 & $\begin{array}{l}125.0- \\
350.0\end{array}$ & $\begin{array}{l}213.0(143.0- \\
272.0)\end{array}$ & $\begin{array}{l}216.0(166.0- \\
279.5)\end{array}$ & $\begin{array}{l}176.0(85.5- \\
244.5)\end{array}$ & 0.145 \\
\hline$<125$ & & $10(17 \%)$ & $2(6 \%)$ & $8(31 \%)$ & 0.012 \\
\hline$\geq 125$ & & 49 (83\%) & $31(94 \%)$ & 18 (69\%) & \\
\hline Ferritin, $\mu \mathrm{g} / \mathrm{L}$ & $\begin{array}{l}30- \\
400\end{array}$ & $\begin{array}{l}534.60 \\
(219.80- \\
1355.63)\end{array}$ & $\begin{array}{l}293.55 \\
(201.90- \\
581.00)\end{array}$ & $\begin{array}{l}1264.20 \\
(577.45- \\
1889.55)\end{array}$ & 0.004 \\
\hline \multicolumn{6}{|c|}{$\begin{array}{l}\text { Data are median (IQR), } \mathrm{n}(\%) \text {, or } \mathrm{n} / \mathrm{N}(\%) \text {, where } \mathrm{N} \text { is the total number of patients with available data. } \mathrm{p} \\
\text { values comparing severe with non-severe patients were calculated by } \chi 2 \text { test, Fisher's exact test, or } \\
\text { Mann-Whitney U test, as appropriated. COVID-19, coronavirus disease } 2019\end{array}$} \\
\hline & & & & & \\
\hline
\end{tabular}




\begin{tabular}{|c|c|c|c|c|c|}
\hline & $\begin{array}{l}\text { Normal } \\
\text { range }\end{array}$ & $\begin{array}{l}\text { All patients } \\
(n=59)\end{array}$ & $\begin{array}{l}\text { Non-severe } \\
\text { patients }(n= \\
33)\end{array}$ & $\begin{array}{l}\text { Severe } \\
\text { patients }(n= \\
26)\end{array}$ & $\begin{array}{l}\mathrm{p} \\
\text { value }\end{array}$ \\
\hline $\begin{array}{l}\text { Erythrocyte sedimentation } \\
\text { rate, } \mathrm{mm} / \mathrm{H}\end{array}$ & $0-15$ & $\begin{array}{l}31.50(15.00- \\
59.75)\end{array}$ & $\begin{array}{l}40.00(16.50- \\
59.50)\end{array}$ & $\begin{array}{l}26.00(15.00- \\
74.00)\end{array}$ & 0.872 \\
\hline D-dimer, $\mu \mathrm{g} / \mathrm{mL}$ & $\leq 0.5$ & $\begin{array}{l}1.73(0.49- \\
2.74)\end{array}$ & $\begin{array}{l}0.65(0.40- \\
2.17)\end{array}$ & $\begin{array}{l}2.69(1.44- \\
8.99)\end{array}$ & 0.001 \\
\hline$\leq 0.5$ & & $14 / 57$ (25\%) & 12/31 (39\%) & $2(8 \%)$ & 0.001 \\
\hline$>0 \cdot 5$ to $\leq 1$ & & $7 / 57(12 \%)$ & $6 / 31(19 \%)$ & $1(4 \%)$ & \\
\hline$>1$ & & $36 / 57$ (63\%) & 13/31 (42\%) & $23(88 \%)$ & \\
\hline Creatine kinase, $\mathrm{U} / \mathrm{L}$ & $\leq 190$ & $\begin{array}{l}85.50 \\
(47.50- \\
196.00)\end{array}$ & $\begin{array}{l}65.00(38.00- \\
162.25)\end{array}$ & $\begin{array}{l}127.00 \\
(60.50- \\
446.50)\end{array}$ & 0.065 \\
\hline $\begin{array}{l}\text { Lactate dehydrogenase, } \\
\mathrm{U} / \mathrm{L}\end{array}$ & $\begin{array}{l}135- \\
225\end{array}$ & $\begin{array}{l}260.00 \\
(223.00- \\
471.00)\end{array}$ & $\begin{array}{l}230.00 \\
(200.00- \\
280.00)\end{array}$ & $\begin{array}{l}471.00 \\
(291.00- \\
628.00)\end{array}$ & 0.000 \\
\hline$\leq 225$ & & $15(25 \%)$ & $14(42 \%)$ & $1(4 \%)$ & 0.001 \\
\hline$>225$ & & $44(75 \%)$ & $19(58 \%)$ & $25(96 \%)$ & \\
\hline $\begin{array}{l}\text { Hypersensitive troponin I, } \\
\mathrm{pg} / \mathrm{mL}\end{array}$ & $\leq 34.2$ & $\begin{array}{l}7.60(3.03- \\
44.70)\end{array}$ & $\begin{array}{l}4.10(1.95- \\
14.15)\end{array}$ & $\begin{array}{l}33.40(4.55- \\
281.85)\end{array}$ & 0.001 \\
\hline$\leq 34.2$ & & 40/54 (74\%) & $27 / 29(93 \%)$ & $13 / 25(52 \%)$ & 0.001 \\
\hline$>34.2$ & & $14 / 54(26 \%)$ & $2 / 29(7 \%)$ & $12 / 25(48 \%)$ & \\
\hline $\begin{array}{l}\text { Alanine aminotransferase, } \\
\mathrm{U} / \mathrm{L}\end{array}$ & $\leq 41$ & $\begin{array}{l}24.00 \\
(15.00- \\
40.00)\end{array}$ & $\begin{array}{l}22.00(14.00- \\
39.50)\end{array}$ & $\begin{array}{l}24.50(17.50- \\
69.00)\end{array}$ & 0.205 \\
\hline$\leq 41$ & & $45(76 \%)$ & $27(82 \%)$ & $18(69 \%)$ & 0.259 \\
\hline$>41$ & & $14(24 \%)$ & $6(18 \%)$ & $8(31 \%)$ & \\
\hline $\begin{array}{l}\text { Aspartate } \\
\text { aminotransferase, U/L }\end{array}$ & $\leq 40$ & $\begin{array}{l}32.00 \\
(19.00- \\
50.00)\end{array}$ & $\begin{array}{l}23.00(18.50- \\
39.50)\end{array}$ & $\begin{array}{l}45.50(21.50- \\
89.25)\end{array}$ & 0.003 \\
\hline$\leq 40$ & & 37 (63\%) & $26(79 \%)$ & $11(42 \%)$ & 0.004 \\
\hline$>40$ & & $22(37 \%)$ & $7(21 \%)$ & $15(58 \%)$ & \\
\hline
\end{tabular}

Data are median (IQR), $\mathrm{n}(\%)$, or $\mathrm{n} / \mathrm{N}(\%)$, where $\mathrm{N}$ is the total number of patients with available data. $\mathrm{p}$ values comparing severe with non-severe patients were calculated by $\chi 2$ test, Fisher's exact test, or Mann-Whitney U test, as appropriated. COVID-19, coronavirus disease 2019

infection. 


\begin{tabular}{|c|c|c|c|c|c|}
\hline & $\begin{array}{l}\text { Normal } \\
\text { range }\end{array}$ & $\begin{array}{l}\text { All patients } \\
(n=59)\end{array}$ & $\begin{array}{l}\text { Non-severe } \\
\text { patients }(n= \\
\text { 33) }\end{array}$ & $\begin{array}{l}\text { Severe } \\
\text { patients }(n= \\
\text { 26) }\end{array}$ & $\begin{array}{l}\mathrm{p} \\
\text { value }\end{array}$ \\
\hline $\begin{array}{l}\text { Blood urea nitrogen, } \\
\mathrm{mmol} / \mathrm{L}\end{array}$ & $\begin{array}{l}3.6- \\
9.5\end{array}$ & $\begin{array}{l}5.10(3.80- \\
8.30)\end{array}$ & $\begin{array}{l}4.70(3.75- \\
5.60)\end{array}$ & $\begin{array}{l}7.90(4.50- \\
15.15)\end{array}$ & 0.004 \\
\hline$\leq 9.5$ & & 47 (80\%) & $32(97 \%)$ & 15 (58\%) & 0.000 \\
\hline$>9.5$ & & $12(20 \%)$ & $1(3 \%)$ & $11(42 \%)$ & \\
\hline Creatinine, $\mu \mathrm{mol} / \mathrm{L}$ & $\begin{array}{l}59- \\
104\end{array}$ & $\begin{array}{l}74.00 \\
(61.00- \\
94.00)\end{array}$ & $\begin{array}{l}72.00(61.00- \\
86.50)\end{array}$ & $\begin{array}{l}80.50(60.00- \\
123.25)\end{array}$ & 0.136 \\
\hline$\leq 104$ & & 49 (83\%) & $32(97 \%)$ & $17(65 \%)$ & 0.001 \\
\hline$>104$ & & $10(17 \%)$ & $1(3 \%)$ & $9(35 \%)$ & \\
\hline Procalcitonin, $\mathrm{ng} / \mathrm{mL}$ & $<0.05$ & $\begin{array}{l}0.08(0.04- \\
0.31)\end{array}$ & $\begin{array}{l}0.04(0.03- \\
0.07)\end{array}$ & $\begin{array}{l}0.18(0.08- \\
1.37)\end{array}$ & 0.000 \\
\hline$<0.05$ & & 18/55 (33\%) & 17/30 (57\%) & $1 / 25(4 \%)$ & 0.000 \\
\hline$\geq 0.05$ & & $37 / 55$ (67\%) & $13 / 30(43 \%)$ & $24 / 25(96 \%)$ & \\
\hline $\begin{array}{l}\text { High-sensitivity C-reactive } \\
\text { Protein (hs-CRP), mg/L }\end{array}$ & $<1$ & $\begin{array}{l}30.55(4.33- \\
124.28)\end{array}$ & $\begin{array}{l}19.85(1.93- \\
34.05)\end{array}$ & $\begin{array}{l}126.15 \\
(27.68- \\
188.43)\end{array}$ & 0.000 \\
\hline$<3$ & & $14 / 58(24 \%)$ & $11 / 32(34 \%)$ & $3(12 \%)$ & 0.043 \\
\hline$\geq 3$ & & $44 / 58(76 \%)$ & $21 / 32(66 \%)$ & $23(88 \%)$ & \\
\hline $\mathrm{IL}-1 \beta, \mathrm{pg} / \mathrm{mL}$ & $<5$ & $\begin{array}{l}5.00(5.00- \\
5.00)\end{array}$ & $\begin{array}{l}5.00(5.00- \\
5.00)\end{array}$ & $\begin{array}{l}5.00(5.00- \\
5.00)\end{array}$ & 0.245 \\
\hline IL-2R, U/L & $\begin{array}{l}223- \\
710\end{array}$ & $\begin{array}{l}645.00 \\
(406.50- \\
1068.00)\end{array}$ & $\begin{array}{l}499.50 \\
(337.25- \\
734.25)\end{array}$ & $\begin{array}{l}973.00 \\
(602.00- \\
1919.00)\end{array}$ & 0.000 \\
\hline $\mathrm{IL}-6, \mathrm{pg} / \mathrm{mL}$ & $<7$ & $\begin{array}{l}11.59(2.34- \\
57.00)\end{array}$ & $\begin{array}{l}5.68(1.83- \\
29.56)\end{array}$ & $\begin{array}{l}47.42(8.64- \\
167.20)\end{array}$ & 0.002 \\
\hline $\mathrm{IL}-8, \mathrm{pg} / \mathrm{mL}$ & $<62$ & $\begin{array}{l}14.40(7.90- \\
47.10)\end{array}$ & $\begin{array}{l}11.20(5.40- \\
21.05)\end{array}$ & $\begin{array}{l}34.10(12.95- \\
81.00)\end{array}$ & 0.001 \\
\hline $\mathrm{IL}-10, \mathrm{pg} / \mathrm{mL}$ & $<9.1$ & $\begin{array}{l}5.00(5.00- \\
7.20)\end{array}$ & $\begin{array}{l}5.00(5.00- \\
5.00)\end{array}$ & $\begin{array}{l}6.30(5.00- \\
19.45)\end{array}$ & 0.001 \\
\hline $\mathrm{TNFa}, \mathrm{pg} / \mathrm{mL}$ & $<8.1$ & $\begin{array}{l}9.20(5.75- \\
12.90)\end{array}$ & $\begin{array}{l}7.65(4.60- \\
10.83)\end{array}$ & $\begin{array}{l}11.80(7.50- \\
17.30)\end{array}$ & 0.021 \\
\hline \multicolumn{6}{|c|}{$\begin{array}{l}\text { Data are median (IQR), } \mathrm{n}(\%) \text {, or } \mathrm{n} / \mathrm{N}(\%) \text {, where } \mathrm{N} \text { is the total number of patients with available data. } \mathrm{p} \\
\text { values comparing severe with non-severe patients were calculated by } \chi 2 \text { test, Fisher's exact test, or } \\
\text { Mann-Whitney U test, as appropriated. COVID-19, coronavirus disease } 2019\end{array}$} \\
\hline
\end{tabular}




\section{Predictors of the severity of COVID-19 patients with chronic airway diseases}

To identify the predictors of the severity of COVID-19 patients with chronic airway diseases, we analyzed the association between the abnormal laboratory findings and the disease severity with univariate and multivariate logistic regression models. The disease severity was significantly associated with all of the above mentioned abnormal laboratory findings in univariate logistic regression analyses. In a multivariate regression model incorporated lymphopenia, eosinopenia, elevated LDH and increased IL-6,

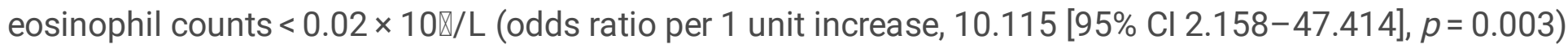
and LDH levels > $225 \mathrm{U} / \mathrm{L}$ (odds ratio per 1 unit increase, 22.300 [2.179-228.247], $p=0.009$ ) were independent risk factors for disease severity (Table 3). Our data suggest that decreased eosinophil counts and increased LDH levels may help the clinician to identify the severe COVID-19 patients with chronic airway diseases. 
Table 3

Predictors of disease severity in COVID-19 patients with chronic airway diseases.

Univariable OR $(95 \% \mathrm{Cl})$

value

Multivariable

OR $(95 \% \mathrm{Cl})$

p value

\section{Laboratory findings}

White blood cell count, $\times 10 \otimes / L$

$1.296(1.087-1.546)$

0.004

$<4$

$0.889(0.223-3.542)$

0.867

4-10

1 (ref)

$>10$

$11.000(2.068-58.519)$

Neutrophil count, $\times 10 \otimes / L$

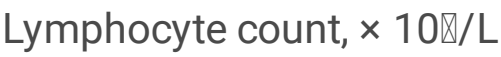

$<1.1$

$\geq 1.1$

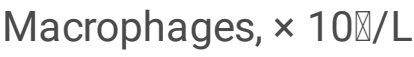

Eosinophil count, $\times 108 / L$ *

$<0.02$

$\geq 0.02$

Basophils, $\times 10 \otimes / L$

1 (ref)

$0.003\left(4.819 \times 10^{-16}\right.$

$1.404 \times 10^{10}$ )

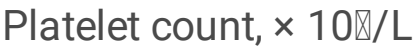

0.996(0.990-1.001)

6.889(1.317-36.042)

1 (ref)

$\geq 125$

1.002(1.000-1.003)

1.001(0.980-1.023)

Erythrocyte sedimentation rate, $\mathrm{mm} / \mathrm{H}$

D-dimer, $\mu \mathrm{g} / \mathrm{mL}$

$\leq 0 \cdot 5$

$>0 \cdot 5$ to $\leq 1$

$\mathrm{OR}=$ odds ratio. ${ }^{*}$ Per 1 unit increase.
$1.305(0.981-1.736)$

1 (ref)

$1.000(0.075-13.367)$
0.005

0.002

0.005

0.006

..

0.839

0.019

..

10.115(2.15847.414)

1 (ref)

0.691

0.117

0.022

..

0.011

0.896

0.067

1.000
..

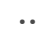

•
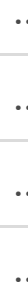


\begin{tabular}{|c|c|c|c|c|}
\hline & Univariable OR (95\% Cl) & $\mathrm{p}_{\text {value }}$ & $\begin{array}{l}\text { Multivariable } \\
\text { OR }(95 \% \mathrm{Cl})\end{array}$ & $\begin{array}{l}\mathrm{p} \\
\text { value }\end{array}$ \\
\hline$>1$ & $10.615(2.051-54.954)$ & 0.005 & .. & .. \\
\hline Creatine kinase, $\mathrm{U} / \mathrm{L}$ & $1.005(0.999-1.010)$ & 0.090 & .. & .. \\
\hline Lactate dehydrogenase, $\mathrm{U} / \mathrm{L}^{*}$ & $1.014(1.006-1.022)$ & 0.000 & .. & .. \\
\hline$\leq 225$ & 1 (ref) & .. & 1 (ref) & .. \\
\hline$>225$ & $18.421(2.223-152.647)$ & 0.007 & $\begin{array}{l}22.300(2.179- \\
228.247)\end{array}$ & 0.009 \\
\hline Hypersensitive troponin I, pg/mL & $1.012(0.998-1.027)$ & 0.085 & .. & .. \\
\hline$\leq 34.2$ & 1 (ref) & .. & .. & .. \\
\hline$>34.2$ & $0.080(0.016-0.412)$ & 0.003 & .. & .. \\
\hline Aspartate aminotransferase, $\mathrm{U} / \mathrm{L}$ & $1.040(1.012-1.069)$ & 0.005 &.. & .. \\
\hline$\leq 40$ & 1 (ref) & .. & .. & .. \\
\hline$>40$ & $5.065(1.618-15.853)$ & 0.005 & .. & .. \\
\hline Blood urea nitrogen, $\mathrm{mmol} / \mathrm{L}$ & $1.269(1.068-1.508)$ & 0.007 & .. & .. \\
\hline$\leq 9.5$ & 1 (ref) & .. & .. & .. \\
\hline$>9.5$ & 23.467(2.769-198.859) & 0.004 & .. & .. \\
\hline Creatinine, $\mu \mathrm{mol} / \mathrm{L}$ & $1.022(1.001-1.044)$ & 0.041 & .. & .. \\
\hline$\leq 104$ & 1 (ref) & .. & .. &. \\
\hline$>104$ & $16.941(1.977-145.160)$ & 0.010 & .. & .. \\
\hline Procalcitonin, ng/mL & $\begin{array}{l}390.345(1.993- \\
76464.041)\end{array}$ & 0.027 & .. & .. \\
\hline$<0.05$ & 1 (ref) & .. & .. & .. \\
\hline$\geq 0.05$ & $31.385(3.742-263.235)$ & 0.001 & .. & .. \\
\hline $\begin{array}{l}\text { High-sensitivity C-reactive Protein } \\
\text { (hs-CRP), mg/L }\end{array}$ & $1.023(1.010-1.036)$ & 0.001 & .. & .. \\
\hline$<3$ & 1 (ref) & .. & .. & .. \\
\hline$\geq 3$ & $4.016(0.983-16.400)$ & 0.053 & .. & .. \\
\hline IL-2R, U/ml & $1.003(1.001-1.004)$ & 0.005 & .. & .. \\
\hline
\end{tabular}

$\mathrm{OR}=$ odds ratio. ${ }^{*}$ Per 1 unit increase. 


\begin{tabular}{|c|c|c|c|c|}
\hline & Univariable OR (95\% Cl) & $p_{\text {value }}$ & $\begin{array}{l}\text { Multivariable } \\
\text { OR (95\% Cl) }\end{array}$ & $\mathrm{p}_{\text {value }}$ \\
\hline IL-6, pg/ml & $1.023(1.003-1.044)$ & 0.022 & .. & .. \\
\hline$<7$ & 1 (ref) & .. & .. & .. \\
\hline$\geq 7$ & $8.000(1.908-33.537)$ & 0.004 & .. & .. \\
\hline IL-8, pg/mL & $1.026(1.002-1.051)$ & 0.030 & .. & .. \\
\hline IL-10, pg/mL & $1.166(0.999-1.363)$ & 0.052 & .. & .. \\
\hline TNF-a, pg/ml & $1.178(1.024-1.355)$ & 0.022 & .. & .. \\
\hline
\end{tabular}

\section{Eosinophil counts and LDH levels tend to return to normal range over time in non-severe patients}

We further analyzed the eosinophil counts and LDH levels in non-severe and severe COVID-19 patients with chronic bronchitis, COPD, and asthma, respectively. We found that there was a significant difference in eosinophil counts and LDH levels between severe and non-severe patients with chronic bronchitis and COPD whereas not in patients with asthma (Fig. 1). To observe the dynamic changes of eosinophil counts and LDH levels over time, we collected the eosinophil counts and LDH levels on the 5th, 10th, 15th, 20th, 25th, and 30th day after admission. We found that eosinophil counts increased over time both in severe and non-severe patients. Meanwhile, LDH decreased over time (Fig. 2). Severe patients showed a slower recovery rate than non-severe patients, especially eosinophil counts. Of note, both eosinophil counts and LDH levels recovered more slowly in severe patients with COPD than those in severe patients with chronic bronchitis and asthma. Our data suggest that, as the disease improved, eosinophil counts and LDH levels tend to return to normal range both in severe and non-severe patients, indicating a good therapeutic effect of patients with chronic airway diseases in COVID-19 treatment.

We further performed multivariate analysis for mortality in COVID-19 patients with chronic airway inflammation using the above four variables and found that eosinophil counts $<0.02 \times 10 \mathbb{Q} / \mathrm{L}$ (odds ratio per 1 unit increase, 18.000 [95\% Cl 1.929-167.986], $p=0.011$ ) was the only independent risk factor for mortality (Supplementary Table 1). Moreover, Kaplan-Meier survival curves indicated that COVID-19 patients with eosinopenia or elevated LDH had worse survival probability $(p<0.05)$ (Supplementary Fig. 1). This suggests that eosinopenia and elevated LDH are also potential predictors for the mortality of COVID-19 patients with underlying chronic airway diseases.

\section{Discussion}

In this retrospective cohort study, we found that eosinophil counts less than $0.02 \times 10 \mathrm{~V} / \mathrm{L}$ and LDH levels greater than $225 \mathrm{U} / \mathrm{L}$ on admission were associated with the severity of COVID patients with underlying chronic bronchitis, COPD and asthma. Moreover, eosinophil counts and LDH levels tend to return to a normal range in severe and non-severe patients after treatment. 
Circulating and tissue-resident eosinophils are associated with a variety of diseases, in which eosinophils participate in the pathological process and play a potent proinflammatory role, such as COPD, asthma, and chronic bronchitis. Previously, human eosinophil has been reported to play an important role in virus detection and defending through several Toll-like receptors (TLRs), including TLR1, TLR3, TLR4, TLR7, TLR9, and TLR10 [18-21]. Single-stranded RNA viruses, such as coronavirus, can be recognized by eosinophils in the airway tract through TLR7, whose subsequent stimulation triggers eosinophil cytokine expression and nitric oxide (NO) generation to promote viral clearance [19-22]. In view of elevated eosinophils in patients with chronic airway inflammation, COPD, asthma and chronic bronchitis have not yet been reported as major risk factors for the severity of SARS-CoV-2 infections. According to an ambispective cohort study of 548 COVID-19 patients, only 5 cases of asthma were identified, significantly lower than previously reported asthma prevalence in Wuhan (6.4\%) [23-26]. Zhang et al recently reported that none had asthma or other comorbid atopic diseases and only two patients had COPD $(1.4 \%)$ in a cohort of 140 hospitalized COVID-19 patients, more than half of whom (53\%) had eosinopenia on the day of hospital admission [23]. Similarly, Du et al analyzed clinical features of 85 fatal cases of COVID-19 and found that $81 \%$ of the patients had very low eosinophil counts on admission [27]. In our cohort including 1888 patients, 31 patients had chronic bronchitis (1.64\%), 18 patients had COPD $(0.95 \%)$ and only 10 patients had asthma ( $0.53 \%)$. Circulating eosinophil counts were reported to gradually increase over the time in COVID-19 and were synchronous with the improvement of chest CT, revealing the effective role of eosinophil in the prognosis monitoring of COVID-19 patients [14]. Liu et al also suggested that elevated eosinophils might be an indicator for COVID-19 improvement in a small cohort of COVID-19 patients [28]. A recent study has highlighted the significant role of $\mathrm{CD}^{101^{-}}$eosinophils in suppressing acute lung injury and respiratory failure [29]. Therefore, eosinophil could have helped patients with chronic airway inflammation escape from SARS-CoV-2 infections and has been identified as a probable potential indicator for prognosis in COVID-19. Jackson et al found a negative correlation between ACE2 expression in airway epithelium and peripheral blood eosinophil counts, which could explain the reason why severe patients were more vulnerable to SARS-CoV-2 infection [30]. Meanwhile, eosinopenia was more common in critically severe patients, suggesting that the resolution of eosinopenia could be a possible way to improve clinical status [31].

In our study, lower expression of eosinophil showed worse survival probability and eosinophil counts significantly decreased in severe COVID-19 patients with chronic bronchitis and COPD. No significant difference was observed in asthma patients, partly due to the limited sample size. We further explored dynamic changes of eosinophil counts in patients with chronic airway diseases in the course of COVID19 and found that eosinophil counts gradually increased over time and returned to normal range in both severe and non-severe patients. It still remains unclear how eosinopenia takes place in COVID-19, but possible mechanisms of decreasing eosinophils could be inhibition of eosinopoiesis and egress of eosinophils from the bone marrow [32,33], the reduction of chemokine receptors or adhesion factors [34], and interferon (IFN) mediated eosinophil apoptosis during the virus infection [33]. 
LDH has long been reported to be associated with COPD, asthma, and chronic bronchitis and identified as a potential marker of chronic airway inflammation [35-37]. Meanwhile, a large number of studies reported elevated LDH levels in COVID-19, which could be a risk factor of mortality [10-12, 38-41]. Zheng et al conducted a systematic literature review and meta-analysis including 4 studies, a total of 1286 cases, and found that LDH was statistically significantly higher in severe patients compared to nonsevere patients [38]. Elevated LDH in severe cases indicated diffuse lung injury and tissue damage [38, 42], therefore, we hypothesized that LDH might be another predictor of chronic airway inflammation exacerbation in COVID-19. Kaplan-Meier survival analysis suggested the hazard of elevated LDH levels. Similar to eosinophil, LDH showed elevated levels in severe COVID-19 patients with chronic bronchitis and COPD, and gradually decreased over time in severe and non-severe COVID-19 patients.

Previous studies have identified older age as a risk factor of mortality in SARS, MERS, and COVID-19 [1012, 43-45]. However, in our study, age had no statistic difference between severe and non-severe patients, partly due to epidemiological characteristic in respiratory diseases with chronic airway inflammation, since such patients were commonly old regardless of disease severity. Lymphocytopenia was also associated with poor outcomes in our cohort (85\%), which is consistent with other reports [40, 46]. Impaired lymphogenesis or increased apoptosis could explain lymphocytopenia in severe cases of COVID-19 [47]. Of note, d-dimer levels greater than $1 \mu \mathrm{g} / \mathrm{L}$ were more common in severe patients compared to non-severe patients, which was reported as a risk factor for mortality of adult inpatients with COVID-19 [10].

Accumulating evidence reveals that cytokine storm plays a crucial role in the pathogenesis of COVID-19. Extremely increased inflammatory parameters, including CRP and proinflammatory cytokines (IL-6, TNFa, IL-8, et al) were recently reported in critical COVID-19 patients [48]. Th1-dominated responses with significantly elevated cytokines (INF- $\gamma, \mathrm{IL}-1 \beta, \mathrm{IL}-6, \mathrm{IL}-8, \mathrm{IL}-12$, and TNF- $\alpha$ ) were shown previously in plasma cytokine profiles of SARS patients, giving rise to the recruitment of alveolar macrophages and the development of ARDS [49-51]. Similarly, a predominant Th1 and Th17 cytokine profile with elevated IFN$y$, TNF-a, IL-10, IL-15, and IL-17 was reported during the acute phase of MERS-CoV infection [52]. In our cohort, severe patients had markedly higher levels of CRP, procalcitonin, IL-2R, IL-6, IL-8, and TNF-a. Notably, several reports confirmed the elevation of serum IL-6 in critically ill patients with COVID-19, suggesting that mortality might be associated with virally driven hyperinflammation and IL-6 played a predominant role in cytokine release syndrome [10, 11, 41, 48, 53-55]. Tocilizumab (IL-6 receptor blockade) has been approved in some patients with COVID-19 pneumonia, offering an effective treatment option for severe patients $[53,56]$.

Our study had some limitations. Firstly, due to the retrospective study design, the accuracy of all laboratory results was dependent upon medical records. Observation bias might also exist in this study due to the limited sample size. Secondly, there could be a selection bias in the multivariate regression model when analyzing the risk factors.

\section{Conclusion}


Our study reveals that eosinopenia and elevated LDH on admission are potential predictors of disease severity in adults with COVID-19 and underlying chronic airway diseases. These predictors may help clinicians identify the severe COVID-19 patients with chronic bronchitis, COPD, and asthma.

\section{Methods}

\section{Study population and data collection}

Subjects of this study were adults with COVID-19 and underlying chronic respiratory diseases (admission date from Jan $26^{\text {th }}$ to April $\left.3^{\text {rd }}, 2020\right)$ in Sino-French New City Branch of Tongji hospital. COVID-19 patients were diagnosed according to World Health Organization (WHO) interim guideline [57]. All patients were confirmed by the positive findings in reverse-transcriptase-polymerase-chain-reaction (RTPCR) assay of SARS-CoV-2 RNA in throat swab specimens. The study was approved by the Institutional Review Board of Tongji Hospital, Huazhong University of Science and Technology.

The demographic information, clinical characteristics (included medical history, symptoms, comorbidities, smoking history, and allergic history) and radiological results of each patient were obtained from the electronic medical record system of Sino-French New City Branch of Tongji hospital and analyzed by three independent researchers. The severity of COVID-19 was staged according to the guidelines for diagnosis and treatment of COVID-19 published by Chinese National Health Committee (Version 5-7).

\section{The criteria for the severity of COVID-19}

Severe COVID-19 patients were diagnosed when conforming to one of the following criteria: 1) Respiratory distress with respiratory frequency $\geq 30 / \mathrm{min} ; 2$ ) Pulse Oximeter Oxygen Saturation $\leq 93 \%$ at rest; 3) Oxygenation index (artery partial pressure of oxygen/inspired oxygen fraction, $\mathrm{PaO2} / \mathrm{FiO} 2$ ) $\leq 300$ $\mathrm{mmHg}$.

\section{Laboratory testing}

Medical laboratory finding results, including the numbers of leucocytes, lymphocytes, monocytes, eosinophils, basophils, platelets, Alanine aminotransferase (ALT), aspartate aminotransferase (AST), serum creatine kinase (CK), serum lactic dehydrogenase (LDH), blood urea nitrogen (BUN), serum creatinine (Scr), cardiac troponin I, concentrations of D-dimer, C-reactive protein (CRP), procalcitonin (PCT), erythrocyte sedimentation rate (ESR), serum ferritin, cytokines (IL-2R, IL-6, IL-8, IL-10, TNF-a), immune function were collected for each patient from electronic medical records.

\section{Statistical analysis}

All data were analyzed with SPSS Statistics Software (version 26; IBM, New York, USA). The statistics for categorical variables were summarized as frequencies and percentages and were compared using Chisquare test or Fisher's exact test between different groups where appropriate. Continuous variables were 
described using median (IQR) and compared using Mann-Whitney $U$ test. To explore the risk factors associated with disease severity, univariable and multivariable logistic regression models were used to estimate odds ratios and the $95 \%$ confidence intervals. A two-sided a of less than 0.05 was considered statistically significant.

\section{Declarations}

\section{Authors' contributions}

LY and GZ conceptualized the study design. LY, DC, SZ, YF, WW, CC, SC collected demographic, clinical, and laboratory data. LY, DC, SZ, YF and GZ analyzed the data. LY and DC interpreted the results. LY, DC and GZ wrote the manuscript with all authors providing feedback for revision. All authors read and approved the final report.

\section{Acknowledgments}

We are sincerely thankful to all front-line members of the medical, nursing, and support staffs of the SinoFrench New City Branch of Tongji Hospital for their support and sacrifice.

\section{Funding}

Supported by National Natural Science Foundation of China (grants 81800026, 81670019, 91742108), National Key Research and Development Program of China (2016YFC1304400).

\section{Ethics approval and consent to participate}

This retrospective study was approved by the institutional ethics board of Tongji Hospital of Tongji Medical College of Huazhong University of Science and Technology. Written informed consent was waived.

\section{Consent for publication}

Not applicable.

\section{Competing interests}

The authors have no competing interests to declare.

\section{References}

1. World Health Organization. Coronavirus disease (COVID-19): Situation Report-146 [https://www.who.int/docs/default-source/coronaviruse/situation-reports/20200614-covid-19-sitrep146.pdf?sfvrsn=5b89bdad_4] 
2. Lu R, Zhao X, Li J, Niu P, Yang B, Wu H, Wang W, Song H, Huang B, Zhu N, et al: Genomic characterisation and epidemiology of 2019 novel coronavirus: implications for virus origins and receptor binding.Lancet (London, England) 2020, 395:565-574.

3. Chan JF-W, Yuan S, Kok K-H, To KK-W, Chu H, Yang J, Xing F, Liu J, Yip CC-Y, Poon RW-S, et al: A familial cluster of pneumonia associated with the 2019 novel coronavirus indicating person-toperson transmission: a study of a family cluster.Lancet (London, England) 2020, 395:514-523.

4. Li Q, Guan X, Wu P, Wang X, Zhou L, Tong Y, Ren R, Leung KSM, Lau EHY, Wong JY, et al: Early Transmission Dynamics in Wuhan, China, of Novel Coronavirus-Infected Pneumonia.New England Journal of Medicine 2020, 382:1199-1207.

5. Barnes PJ: Targeting cytokines to treat asthma and chronic obstructive pulmonary disease. Nature Reviews Immunology 2018, 18:454-466.

6. Rabe KF, Watz H: Chronic obstructive pulmonary disease. The Lancet 2017, 389:1931-1940.

7. Papi A, Brightling C, Pedersen SE, Reddel HK: Asthma.The Lancet 2018, 391:783-800.

8. Lambrecht BN, Hammad H, Fahy JV: The Cytokines of Asthma.Immunity 2019, 50:975-991.

9. Kim V, Criner GJ: Chronic Bronchitis and Chronic Obstructive Pulmonary Disease.American Journal of Respiratory and Critical Care Medicine 2013, 187:228-237.

10. Zhou F, Yu T, Du R, Fan G, Liu Y, Liu Z, Xiang J, Wang Y, Song B, Gu X, et al: Clinical course and risk factors for mortality of adult inpatients with COVID-19 in Wuhan, China: a retrospective cohort study. The Lancet 2020, 395:1054-1062.

11. Wu C, Chen X, Cai Y, Xia Ja, Zhou X, Xu S, Huang H, Zhang L, Zhou X, Du C, et al: Risk Factors Associated With Acute Respiratory Distress Syndrome and Death in Patients With Coronavirus Disease 2019 Pneumonia in Wuhan, China.JAMA internal medicine 2020.

12. Wu Z, McGoogan JM: Characteristics of and Important Lessons From the Coronavirus Disease 2019 (COVID-19) Outbreak in China: Summary of a Report of 72314 Cases From the Chinese Center for Disease Control and Prevention.JAMA 2020, 323:1239-1242.

13. Li H, Liu L, Zhang D, Xu J, Dai H, Tang N, Su X, Cao B: SARS-CoV-2 and viral sepsis: observations and hypotheses. The Lancet 2020, 395:1517-1520.

14. Xie G, Ding F, Han L, Yin D, Lu H, Zhang M: The role of peripheral blood eosinophil counts in COVID19 patients.Allergy 2020, n/a.

15. Liu W, Tao Z-W, Wang L, Yuan M-L, Liu K, Zhou L, Wei S, Deng Y, Liu J, Liu H-G, et al: Analysis of factors associated with disease outcomes in hospitalized patients with 2019 novel coronavirus disease.Chinese medical journal 2020, 133:1032-1038.

16. Li K, Wu J, Wu F, Guo D, Chen L, Fang Z, Li C: The Clinical and Chest CT Features Associated With Severe and Critical COVID-19 Pneumonia.Investigative radiology 2020, 55:327-331.

17. Rod JE, Oviedo-Trespalacios O, Cortes-Ramirez J: A brief-review of the risk factors for covid-19 severity.Revista de saude publica 2020, 54:60. 
18. Månsson A, Fransson M, Adner M, Benson M, Uddman R, Björnsson S, Cardell LO: TLR3 in Human Eosinophils: Functional Effects and Decreased Expression during Allergic Rhinitis.International Archives of Allergy and Immunology 2010, 151:118-128.

19. Nagase H, Okugawa S, Ota Y, Yamaguchi M, Tomizawa H, Matsushima K, Ohta K, Yamamoto K, Hirai K: Expression and Function of Toll-Like Receptors in Eosinophils: Activation by Toll-Like Receptor 7 Ligand. The Journal of Immunology 2003, 171:3977.

20. Wong CK, Cheung PFY, Ip WK, Lam CWK: Intracellular Signaling Mechanisms Regulating Toll-Like Receptor-Mediated Activation of Eosinophils.American Journal of Respiratory Cell and Molecular Biology 2007, 37:85-96.

21. Drake MG, Bivins-Smith ER, Proskocil BJ, Nie Z, Scott GD, Lee JJ, Lee NA, Fryer AD, Jacoby DB: Human and Mouse Eosinophils Have Antiviral Activity against Parainfluenza Virus.American Journal of Respiratory Cell and Molecular Biology 2016, 55:387-394.

22. Edwards MR, Strong K, Cameron A, Walton RP, Jackson DJ, Johnston SL: Viral infections in allergy and immunology: How allergic inflammation influences viral infections and illness.Journal of Allergy and Clinical Immunology 2017, 140:909-920.

23. Zhang J-j, Dong X, Cao Y-y, Yuan Y-d, Yang Y-b, Yan Y-q, Akdis CA, Gao Y-d: Clinical characteristics of 140 patients infected with SARS-CoV-2 in Wuhan, China.Allergy 2020, n/a.

24. Huang K, Yang T, Xu J, Yang L, Zhao J, Zhang X, Bai C, Kang J, Ran P, Shen H, et al: Prevalence, risk factors, and management of asthma in China: a national cross-sectional study. The Lancet 2019, 394:407-418.

25. Wang XD, Zheng M, Lou HF, Wang CS, Zhang Y, Bo MY, Ge SQ, Zhang N, Zhang L, Bachert C: An increased prevalence of self-reported allergic rhinitis in major Chinese cities from 2005 to 2011.Allergy 2016, 71:1170-1180.

26. Li X, Xu S, Yu M, Wang K, Tao Y, Zhou Y, Shi J, Zhou M, Wu B, Yang Z, et al: Risk factors for severity and mortality in adult COVID-19 inpatients in Wuhan.Journal of Allergy and Clinical Immunology 2020.

27. Du Y, Tu L, Zhu P, Mu M, Wang R, Yang P, Wang X, Hu C, Ping R, Hu P, et al: Clinical Features of 85 Fatal Cases of COVID-19 from Wuhan. A Retrospective Observational Study.American Journal of Respiratory and Critical Care Medicine 2020, 201:1372-1379.

28. Liu F, Xu A, Zhang Y, Xuan W, Yan T, Pan K, Yu W, Zhang J: Patients of CoVID-19 may benefit from sustained Lopinavir-combined regimen and the increase of Eosinophil may predict the outcome of COVID-19 progression.International Journal of Infectious Diseases 2020, 95:183-191.

29. Zhu C, Weng Q-Y, Zhou L-R, Cao C, Li F, Wu Y-F, Wu Y-P, Li M, Hu Y, Shen J-X, et al: Homeostatic and Early Recruited CD101 Eosinophils Suppress Endotoxin-induced Acute Lung Injury. The European respiratory journal 2020.

30. Jackson DJ, Busse WW, Bacharier LB, Kattan M, O'Connor GT, Wood RA, Visness CM, Durham SR, Larson D, Esnault S, et al: Association of respiratory allergy, asthma, and expression of the SARSCoV-2 receptor ACE2.Journal of Allergy and Clinical Immunology 2020, 146:203-206.e203. 
31. Lindsley AW, Schwartz JT, Rothenberg ME: Eosinophil responses during COVID-19 infections and coronavirus vaccination.Journal of Allergy and Clinical Immunology 2020.

32. Bass DA: Behavior of eosinophil leukocytes in acute inflammation. II. Eosinophil dynamics during acute inflammation. The Journal of Clinical Investigation 1975, 56:870-879.

33. Butterfield JH: Treatment of hypereosinophilic syndromes with prednisone, hydroxyurea, and interferon.Immunology and allergy clinics of North America 2007, 27:493-518.

34. Hassani M, Leijte G, Bruse N, Kox M, Pickkers P, Vrisekoop N, Koenderman L: Differentiation and activation of eosinophils in the human bone marrow during experimental human endotoxemia.Journal of Leukocyte Biology 2020, n/a.

35. Faruqi S, Wilmot R, Wright $C$, Morice AH: Serum LDH in chronic cough: a potential marker of airway inflammation. The Clinical Respiratory Journal 2012, 6:81-87.

36. Nillawar A, Bardapurkar J, Bardapurkar S: High sensitive C-reactive protein as a systemic inflammatory marker and LDH-3 isoenzyme in chronic obstructive pulmonary disease.Lung India 2012, 29:24-29.

37. Loppow D, Schleiss MB, Kanniess F, Taube C, JÖRres RA, Magnussen H: In patients with chronic bronchitis a four week trial with inhaled steroids does not attenuate airway inflammation.Respiratory Medicine 2001, 95:115-121.

38. Zheng Z, Peng F, Xu B, Zhao J, Liu H, Peng J, Li Q, Jiang C, Zhou Y, Liu S, et al: Risk factors of critical \& mortal COVID-19 cases: A systematic literature review and meta-analysis. Journal of Infection 2020.

39. Huang C, Wang Y, Li X, Ren L, Zhao J, Hu Y, Zhang L, Fan G, Xu J, Gu X, et al: Clinical features of patients infected with 2019 novel coronavirus in Wuhan, China.Lancet (London, England) 2020, 395:497-506.

40. Wang D, Hu B, Hu C, Zhu F, Liu X, Zhang J, Wang B, Xiang H, Cheng Z, Xiong Y, et al: Clinical Characteristics of 138 Hospitalized Patients With 2019 Novel Coronavirus-Infected Pneumonia in Wuhan, China.JAMA 2020, 323:1061-1069.

41. Chen N, Zhou M, Dong X, Qu J, Gong F, Han Y, Qiu Y, Wang J, Liu Y, Wei Y, et al: Epidemiological and clinical characteristics of 99 cases of 2019 novel coronavirus pneumonia in Wuhan, China: a descriptive study.Lancet (London, England) 2020, 395:507-513.

42. Xu Z, Shi L, Wang Y, Zhang J, Huang L, Zhang C, Liu S, Zhao P, Liu H, Zhu L, et al: Pathological findings of COVID-19 associated with acute respiratory distress syndrome. The Lancet Respiratory Medicine 2020, 8:420-422.

43. Lee N, Hui D, Wu A, Chan P, Cameron P, Joynt GM, Ahuja A, Yung MY, Leung CB, To KF, et al: A Major Outbreak of Severe Acute Respiratory Syndrome in Hong Kong.New England Journal of Medicine 2003, 348:1986-1994.

44. Hong K-H, Choi J-P, Hong S-H, Lee J, Kwon J-S, Kim S-M, Park SY, Rhee J-Y, Kim B-N, Choi HJ, et al: Predictors of mortality in Middle East respiratory syndrome (MERS). Thorax 2018, 73:286. 
45. Peiris JSM, Yuen KY, Osterhaus ADME, Stöhr K: The Severe Acute Respiratory Syndrome.New England Journal of Medicine 2003, 349:2431-2441.

46. Guan W-j, Ni Z-y, Hu Y, Liang W-h, Ou C-q, He J-x, Liu L, Shan H, Lei C-I, Hui DSC, et al: Clinical Characteristics of Coronavirus Disease 2019 in China.New England Journal of Medicine 2020, 382:1708-1720.

47. Marshall JC, Charbonney E, Gonzalez PD: The Immune System in Critical Illness. Clinics in Chest Medicine 2008, 29:605-616.

48. Zhang W, Zhao Y, Zhang F, Wang Q, Li T, Liu Z, Wang J, Qin Y, Zhang X, Yan X, et al: The use of antiinflammatory drugs in the treatment of people with severe coronavirus disease 2019 (COVID-19): The Perspectives of clinical immunologists from China.Clinical immunology (Orlando, Fla) 2020, 214:108393.

49. Sheng W-H, Chiang B-L, Chang S-C, Ho H-N, Wang J-T, Chen Y-C, Hsiao C-H, Hseuh P-R, Chie W-C, Yang P-C: Clinical manifestations and inflammatory cytokine responses in patients with severe acute respiratory syndrome.Journal of the Formosan Medical Association = Taiwan yi zhi 2005, 104:715723.

50. Zhu M: SARS Immunity and Vaccination.Cellular \& molecular immunology 2004, 1:193-198.

51. Wong CK, Lam CWK, Wu AKL, Ip WK, Lee NLS, Chan IHS, Lit LCW, Hui DSC, Chan MHM, Chung SSC, Sung JJY: Plasma inflammatory cytokines and chemokines in severe acute respiratory syndrome.Clinical \& Experimental Immunology 2004, 136:95-103.

52. Mahallawi WH, Khabour OF, Zhang Q, Makhdoum HM, Suliman BA: MERS-CoV infection in humans is associated with a pro-inflammatory Th1 and Th17 cytokine profile.Cytokine 2018, 104:8-13.

53. Luo P, Liu Y, Qiu L, Liu X, Liu D, Li J: Tocilizumab treatment in COVID-19: A single center experience.Journal of Medical Virology 2020, 92:814-818.

54. Mehta P, McAuley DF, Brown M, Sanchez E, Tattersall RS, Manson JJ: COVID-19: consider cytokine storm syndromes and immunosuppression. The Lancet 2020, 395:1033-1034.

55. Ruan Q, Yang K, Wang W, Jiang L, Song J: Clinical predictors of mortality due to COVID-19 based on an analysis of data of 150 patients from Wuhan, China.Intensive care medicine 2020, 46:846-848.

56. Zhang C, Wu Z, Li J-W, Zhao H, Wang G-Q: Cytokine release syndrome in severe COVID-19: interleukin-6 receptor antagonist tocilizumab may be the key to reduce mortality.International Journal of Antimicrobial Agents 2020, 55:105954.

57. World Health Organization. Clinical management of severe acute respiratory infection when novel coronavirus (2019-nCoV) infection is suspected: interim guidance [https://www.who.int/docs/default-source/coronaviruse/clinical-management-of-novel-cov.pdf]

\section{Figures}


A

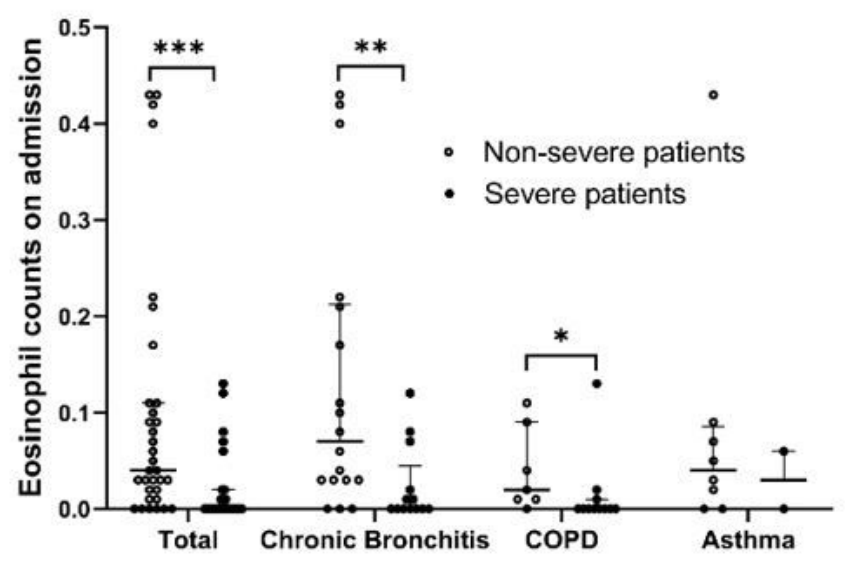

B

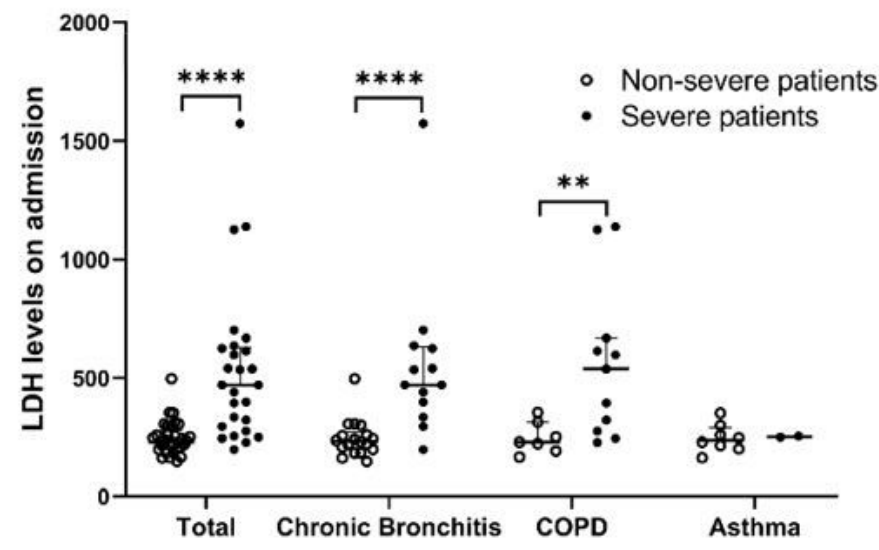

\section{Figure 1}

Clinical characteristics of eosinophil and LDH in COVID-19 patients with chronic airway inflammation. (A) Eosinophil counts in different subgroups. Eosinophil counts were significantly decreased in severe COVID-19 patients with chronic bronchitis and COPD; (B) LDH levels in different subgroups. LDH levels were significantly decreased in severe COVID-19 patients with chronic bronchitis and COPD. Values of non-severe and severe patients were presented with open and closed circles, respectively. Mann-Whitney U test was used. ${ }^{*} p<0.05,{ }^{* *} p<0.01,{ }^{* *} p<0.001, * * \star * p<0.0001$

A

All Patients

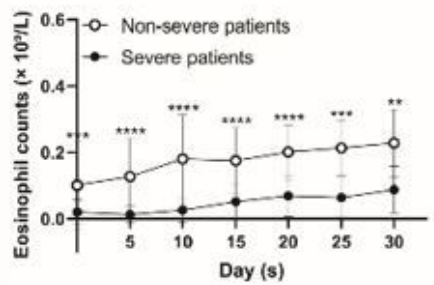

$E$

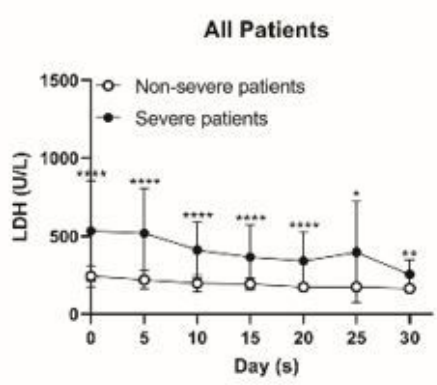

B

Patients with Chronic Bronchitis

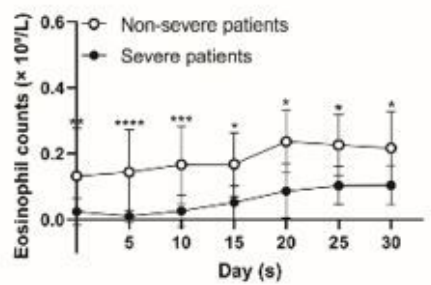

F

Patients with Chronic Bronchitis

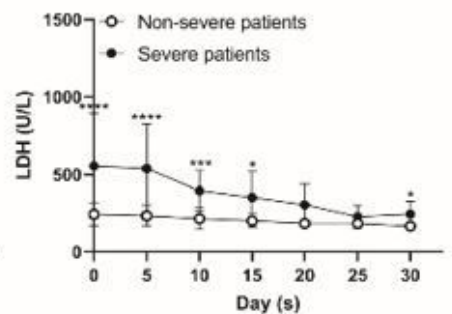

C

Patients with COPD

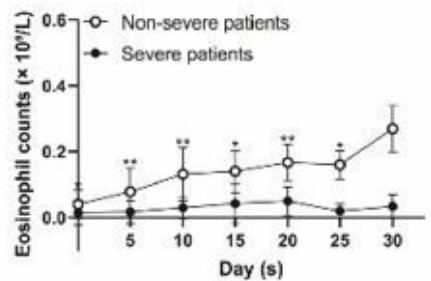

G

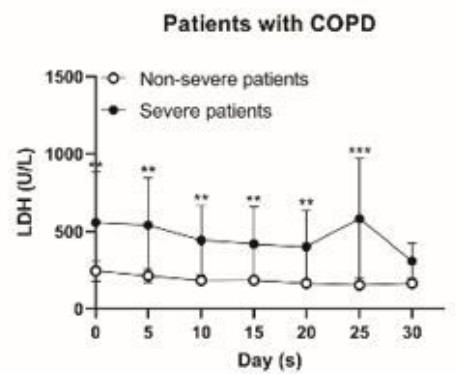

D

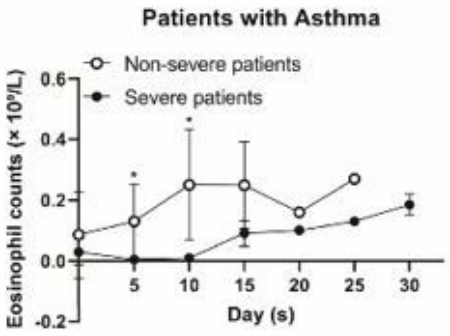

$\mathrm{H}$

Patients with Asthma

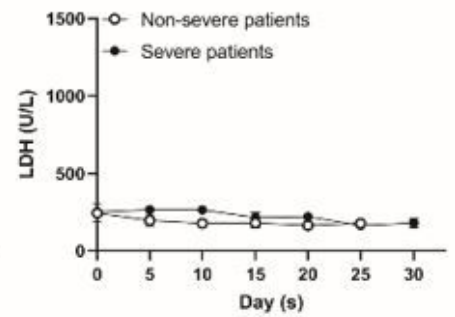

Figure 2 
Dynamic changes of eosinophil counts and LDH levels in COVID-19 patients with chronic airway diseases. (A-D) Eosinophil counts increased over time in non-severe and severe COVID-19 patients with chronic bronchitis $(n=31), \operatorname{COPD}(n=18)$ and asthma $(n=10)$. (E-H) LDH levels decreased over time in non-severe and severe COVID-19 patients with chronic bronchitis $(n=31), \operatorname{COPD}(n=18)$ and asthma $(n=$ 10). Values of non-severe and severe patients were presented with open and closed circles, respectively. Mann-Whitney U test was used. ${ }^{\star} p<0.05,{ }^{\star \star} p<0.01$, ${ }^{\star \star \star} p<0.001$, ${ }^{\star \star \star \star} p<0.0001$, compared with the eosinophil counts or LDH levels between severe and non-severe patients.

\section{Supplementary Files}

This is a list of supplementary files associated with this preprint. Click to download.

- Supplymentarydata.pdf 